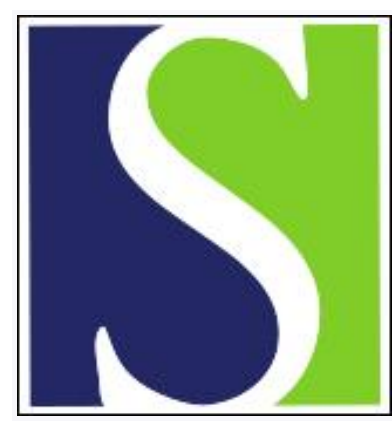

Scand J Work Environ Health 2003;29(2):83-84

https://doi.org/10.5271/sjweh.708

Issue date: Apr 2003

Is the life expectancy of anesthesiologists decreased?

by Hagmar L

Affiliation: Department of Occupational and Environmental Medicine, Lund University Hospital, SE-22185 Lund Sweden. lars.hagmar@ymed.lu.se

Refers to the following text of the Journal: 2001;27(3):157-160

Key terms: anesthesiologist; editorial; life expectancy

This article in PubMed: www.ncbi.nlm.nih.gov/pubmed/12718492

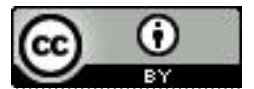




\section{Is the life expectancy of anesthesiologists decreased?}

At an anesthesiology meeting held in August 2000, results were presented claiming that Swedish anesthesiologists had increased mortality when compared with other medical specialists. This presentation caused big headlines in the news media, and there were expressions of profound concern, not to mention anxiety, from anesthesiologists in both Sweden and other countries. Unfortunately, the results of the Swedish study were not published until very recently (1). In the same issue of Acta Anaesthesiologica Scandinavica, similar studies from Denmark, Finland, and Norway also appeared (2-4).

The story about the potentially risky life of anesthesiologists did not start, however, with the Swedish study. In 1996, two British bacteriologists claimed that the mean age of death was lower for anesthesiologists than for other physicians (5). Their analysis was based on 572 obituaries reported in the British Medical Journal. The study gave an example of a rather elementary fallacy in survival studies and was also therefore strongly criticized $(6,7)$. At the end of follow-up in survival studies, the event (in this case death) will probably not have occurred for all subjects. We do not know when the subjects will die, only that they have not done so by the end of the observation period. If the age distributions differ between two populations, it is obvious that the mean age of death during a limited observation period is lower for the younger group than for the older one. This phenomenon does not provide any indication, however, of whether the life expectancies differ between the two populations. This was exactly the problem with the British study, as the anesthesiologists, as a group, were younger than the physicians in other specialties. Thus, due to its methodological flaws, the British study (5) did not provide any meaningful information on the life expectancy of anesthesiologists.

In a large and seemingly well-done American study, the cause-specific mortality between 1979 and 1995 for about 40000 anesthesiologists was compared with that of a gender- and age-comparable sample of internists (8). The all-cause mortality ratios, and the risks of death caused by cancer and heart disease, did not differ between the anesthesiologists and internists, but the anesthesiologists had increased risks of death from cerebrovascular disease, suicide, drug abuse, and other external causes. The authors concluded that substance abuse and suicide represent significant occupational hazards for anesthesiologists.

The results of the Nordic studies were highly inconsistent. After adjustment for age and gender in a Cox regression model, in Sweden a 46\% increase in overall mortality was observed for anesthesiologists as compared with other specialists (1). The study population consisted of all physicians who were specialists in 1993 or had become specialists during the period 1993-1999.

In contrast to the Swedish results, no increased risks were found in the Danish, Norwegian, or Finnish studies (2-4). Both the Norwegian and Finnish studies were, however, rather small and also hampered by methodological weaknesses $(3,4)$.

The Norwegian study was performed as a survival analysis of 10367 Norwegian physicians who, in 1990, were alive and specialists or who were alive and became specialists during the period 1990-2001 (4). The starting year of the survival analysis was not given, but it ought to have been 1990. Accumulation of person-years before 1990 would, of course, be wrong, because no deaths in the cohort could have occurred before that calendar year, as a criterion for inclusion was to be alive in 1990. The author stated, however, that altogether 171190 person-years under risk were observed. This high number clearly shows that person-years must have been accumulated already before 1990. This fallacy makes it impossible to draw any meaningful conclusions from the study.

The results from the small Finnish study were based on descriptive statistics, and no incidence calculations were based on person-years (3). Not much weight can therefore be given to the results of this study. 
The Danish study population consisted of all physicians who were specialists in 1973 or who became specialists during the period 1973-1992 (2). Cox regression models, adjusting for the potential confounders gender, age, year of birth, and whether becoming a specialist before or after 1973 , showed that anesthesiologists did not differ in relative overall mortality risk $(1.02,95 \%$ confidence interval 0.74-1.40) as compared with other specialists.

The authors of the Swedish study considered chance as an unlikely explanation for their finding of an increased overall mortality risk for anesthesiologists and argued that there were "... similar findings in the UK and in the USA with even larger study populations [p 1193]" (1). This line of argumentation is hard to follow. The British study (5) was flawed and impossible to interpret, and the study from the United States (8) did not support the Swedish findings of overall excess mortality.

If the Swedish data hold true, the following three pertinent questions are without any current answers:

1. For which specific causes of death did the anesthesiologists have increased risks?

2. Is it reasonable to assume that the work conditions for Swedish anesthesiologists have been much worse than for anesthesiologists in the United States or Denmark?

3. Is it probable that a larger fraction of Swedish physicians, than of their Danish or American colleagues, with unfavorable personality traits or life-style habits has chosen to become anesthesiologists?

From the point of view of deeper analyses of causes and effects, cause-specific mortality gives more information on what preventive action should be taken than total mortality does (9). Thus, in order to respond to the first question, cause-specific mortality analyses should be performed, even if the statistical power of the Swedish study is still limited. Analyses with respect to duration of work as an anesthesiologist could provide clues for answering the two last questions. If personal traits or life-style habits have been important contributors to the increased mortality, risk enhancement can have been expected to occur already during the first few years after the physicians became specialists in anesthesiology. On the other hand, if work conditions have been hazardous, it is reasonable to expect that excess mortality will primarily not be apparent until later, after work as a specialist has begun.

Until more is known, it seems unwarranted to base preventive action plans for anesthesiologists on the contradictory epidemiologic findings. There may, however, be other health-based reasons for decreasing the workload and stress of anesthesiologists.

\section{References}

1. Svärdsudd K, Wedel H, Gordh T. Mortality rates among Swedish physicians: a population-based nationwide study with special reference to anesthesiologists. Acta Anaesthesiol Scand 2002;46:1187-95.

2. Juel K, Husum B, Viby-Mogensen J, Viskum S. Mortality among anesthesiologists in Denmark, 1973-95. Acta Anaesthesiol Scand 2002;46:1203-05.

3. Ohtonen P, Alahuhta S. Mortality among Finnish anesthesiologists from 1984-2000. Acta Anaesthesiol Scand 2002;6: 196-9.

4. Aasland OG. Mortality of anesthesiologists, pediatricians, and other specialists in Norway. Acta Anaesthesiol Scand 2002;46:1200-2.

5. Wright DJM, Roberts AP. Which doctors die first? Analysis of BMJ obituary columns. BMJ 1996;313:1581-2.

6. Khaw K-T. Which doctors die first? BMJ 1997;314:1132.

7. McManus C. Which doctors die first? Recording the doctors' sex might have led authors to suspect their conclusions. BMJ 1997;314:1132.

8. Alexander BH, Checkoway H, Nagahama SI, Domino KB. Cause-specific mortality risks of anesthesiologists. Anesthesiology 2000;93:922-30.

9. Hernberg S. Work-related factors and mortality—what is the burden? Scand J Work Environ Health 2001;27:157-60.

\section{Lars Hagmar}

Department of Occupational and Environmental Medicine

Lund University Hospital

SE-22185 Lund Sweden

[E-mail: lars.hagmar@ymed.Iu.se] 